\title{
Chaos Communication System using MIMO Technique
}

\author{
Sung-Wook Yoon, Jun-Hyun Lee, and Heung-Gyoon Ryu \\ Department of Electronics and Engineering, Chungbuk National University, Cheongju, Chungbuk, 361-763, Korea \\ magi11@naver.com,toogee89@nate.com, ecomm@cbu.ac.kr
}

\begin{abstract}
The security of chaos communication system is superior to other digital communication system, because it has characteristics such as non-periodic, wide-band, nonpredictability, easy implementation and sensitive initial condition. However, chaos communication system increases the number of transmitted symbols by spreading and transmitting information bits according to characteristic of chaos maps. So the research that improves data transmission speed is necessary for chaos communication system. If many antennas are applied to chaos communication system, the capacity of data is proportional to the number of antenna.so it is good way applying multiple-input and multiple-output (MIMO) to the chaos communication system. In this paper, we propose the correlation delay shift keying (CDSK) using $2 \times 2$ MIMO technique and evaluate BER performance over Rayleigh MIMO fading channel. Lastly we evaluate BER performance applying the boss map with $2 \times 2$ MIMO using MIMO detection algorithm such as zero forcing (ZF) and minimum mean square error (MMSE).
\end{abstract}

Keywords-Chaos Communication System; CDSK; MIMO; Zero Forcing; MMSE;

\section{Introduction}

Previous digital communication technology continually used a linear system. However, as this technology reached basic limit, people started to improve performance of nonlinear communication systems applying chaos communication systems to nonlinear systems [1]. Chaos communication systems have the characteristics such as nonperiodic, wide-band, non-predictability and easy implementation. Also, chaos communication system is decided by initial conditions of equation, and it has sensitive characteristic according to initial condition, because chaos signal is changed to different signal when initial condition is changed [2-3]. Chaos signal is expressed as randomly and non-linearly generated signal, but it is possible to predict in principle. And it is possible to determine value of the future by value of the past. So, if initial conditions of chaos signal is not exact, users of chaos system are impossible to predict the value of chaos signal because chaos signal has sensitive characteristic about initial conditions [1][4]. As these characteristics, the security of chaos communication system is superior to digital communication system.

Due to security and advantage, chaos communication system are being studied continuously. Look at existing research, in order to solve disadvantage that bit error rate
(BER) performance of this system is bad, chaos communication system is evaluated the BER performance according to chaos maps, and find a chaos map that has the best BER performance [5]. In addition, chaos users evaluate the BER performance according to chaos modulation system [6-7], and propose a new chaos map that has the best BER performance according to analysis of probability density function[8]. Also, studies about security and anti-jamming of chaos communication system was studied [9-10].

Chaos communication system has many transmitted symbols, because information signal is spread according to the characteristic of chaos map. So, study of chaos system for data rate improvement is necessary. MIMO(Multi Input Multi Output) system transmit data by several paths using several antennas. And, in receiver part, it is a technique which can reduce interference by detecting received signal at each path, and make better each data rate. MIMO system increase the capacity of data communication in proportion to number of antenna, and provide the high data processing Speed[11-12]. Therefore, study that MIMO system is applied to chaos communication system is important. MIMO detection algorithm is necessary in receiver part when MIMO system is used because signal that transmit from different antenna is added to desired signal. Also, each MIMO detection algorithm is necessary to evaluate and find MIMO detection algorithm that has good performance, because BER performance is different according to MIMO detection algorithm

In this paper, in Rayleigh fading channel, BER performances of CDSK system that is applied 2x2 MIMO with Zero Forcing and MMSE evaluate. Also, BER performances of CDSK system that is applied 2x2 MIMO with MIMO detection algorithm is evaluated, and we compare BER performance of Zero Forcing and MMSE. At existing study, we proposed a novel chaos map in order to improve the BER performance[8], and we named a novel chaos map "Boss map". And we evaluate the performance of Zero Forcing and MMSE when $2 \times 2$ MIMO is applied to CDSK using Boss map.

\section{System Overview}

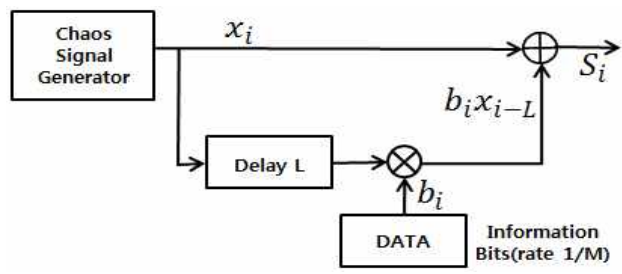

Fig. 1. Transmitter of CDSK system. 


\section{A. Correlation Delay Shift Keying system}

CDSK system has a adder in transmitter. Existing modulation system than CDSK system consists switch in transmitter, and problem of power waste and eavesdropping occurs by twice transmission. Technique that has been proposed for these problems solution is CDSK system. And, transmitted signal does not repeat by replacing an adder with a switch in the transmitter.

Figure 1 shows transmitter of CDSK system. CDSK transmitter is composed of sum that is added delay chaos signal multiplied information bit and generated chaos signal from chaos signal generator. Also, $\mathrm{M}$ means spreading factor, and information bit that is spread as much as spreading factor is multiplied by delay chaos signal.

$$
S_{i}=x_{i}+b_{i} x_{i-L}
$$

Equation(1) indicates transmitted signal from transmitter. Also, L means delay time, and this equation is expressed as sum that is added delay chaos signal multiplied information bit and generated chaos signal.

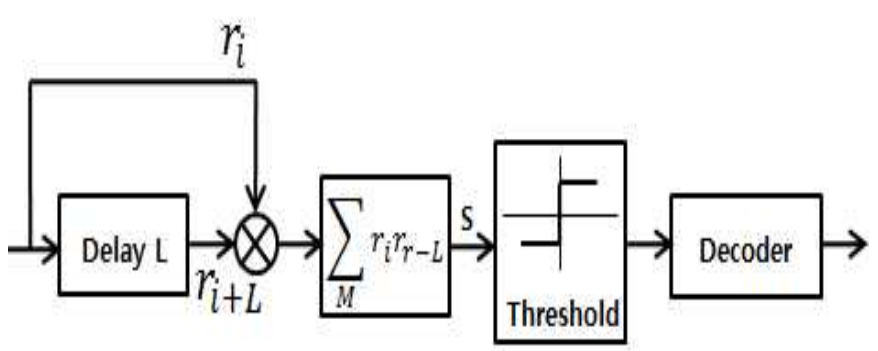

Fig. 2. Receiver of CDSK system.

Figure 2 shows receiver of CDSK system. CDSK receiver is correlator based receiver, and it is performed in order to recover the symbol. Received signal and delay received signal are multiplied, and this signal is as much added as spreading factor. Afterward the signal pass through the threshold, and information signal recover through decoding.

$$
S=\sum_{i=1}^{M} r_{i} r_{i-L}
$$

Equation(2) indicates output value of correlator of CDSK system. At this time, information bits is possible to recover when delay time(L) and spreading factor(M) have to use exact value that is used in transmitted signal.

\section{B. Chaos map}

In this paper, types of chaos map are used Tent map and Boss map. At existing study, Boss map means a novel chaos that we proposed for BER performance improvement[8].

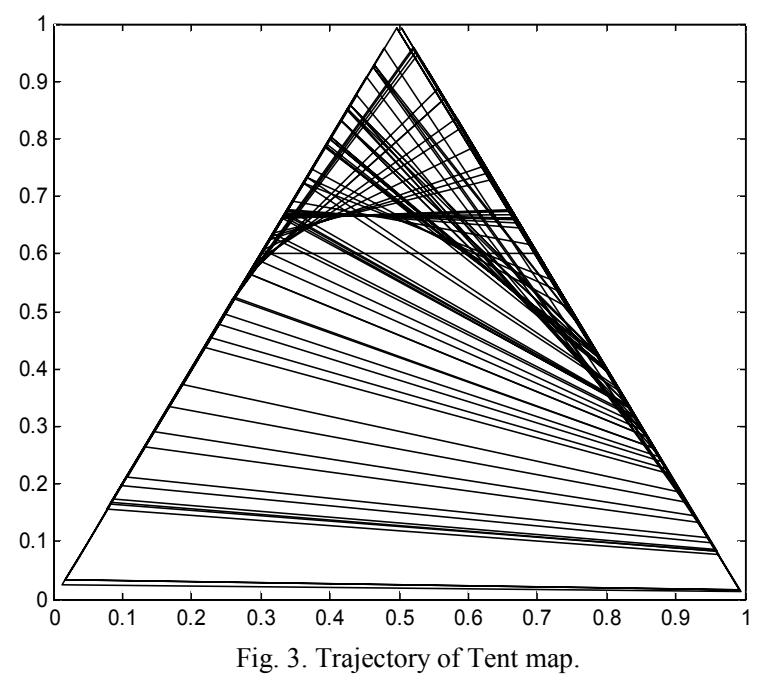

Figure 3 shows trajectory of Tent map. The x-axis and the $\mathrm{y}$-axis of figure 3 mean $x_{n}$ and $x_{n+1}$, and Tent map has trajectory of triangular shape.

$$
x_{n+1}=\left\{\begin{array}{ccc}
\alpha x_{n} & \text { for } & x_{n}<0.5 \\
\alpha\left(1-x_{n}\right) & \text { for } & 0.5 \leq x_{n}
\end{array}\right.
$$

Equation of Tent map is expressed as equation(3). Equation of Tent map uses existing output value as current input value, and it is indicated as figure 3 when initial value is 0.1 and parameter alpha is 1.9999 .

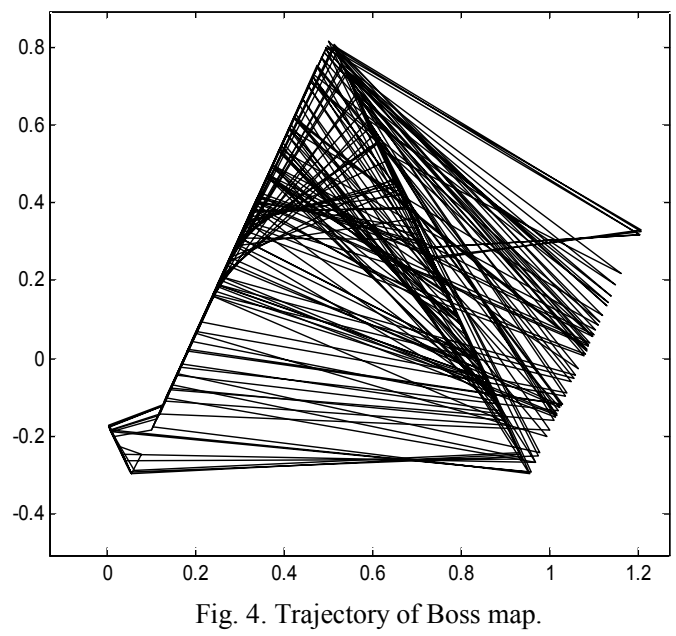

Figure 4 shows trajectory of Boss map that proposed a novel chaos map in order to improve the BER performance. The $\mathrm{x}$-axis and the $\mathrm{y}$-axis of Boss map mean $x_{n}$ and $y_{n}$ unlike the Tent map, it draws trajectory like pyramid shape. 


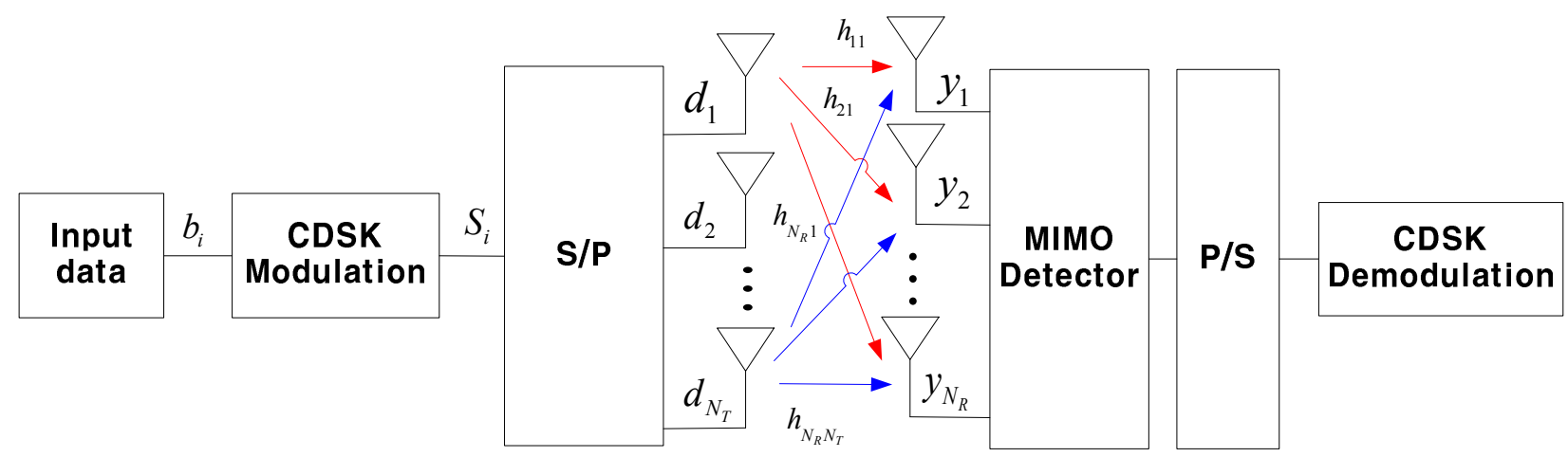

Fig. 5. Block diagram of CDSK system with 2x2 MIMO.

$$
\begin{aligned}
& x_{n+1}=\alpha|0.45-| 0.503-x_{n}|| \\
& y_{n+1}=x_{n}-0.3
\end{aligned}
$$

Equation of Boss map is expressed as equation(4). Equation form of Boss map is similar to Tent map because Boss map was proposed by transforming from Tent map. And, trajectory of Boss map is indicated as figure 4 when initial value is 0.1 and parameter alpha is 2.5 .

\section{CDSK System applied MIMO Technique}

In order to apply the MIMO system to other systems, MIMO detection algorithm is necessary. Types of MIMO detection algorithm are various, but we apply Zero Forcing and MMSE in this paper. Also, performance evaluation of each algorithm is required because BER performance is changed according to types of MIMO detection algorithm.

Figure 5 shows the block diagram of CDSK system that applied 2x2 MIMO system. In MIMO environment, received signal includes transmitted signal from the others antenna except desired signal. So, MIMO detection algorithm is used in order to detach this signal. Afterward, information bits are recovered through CDSK receiver. We assume that the number of transmitter and receiver antenna is 2 .

$$
\begin{aligned}
& y_{1}=h_{1,1} d_{1}+h_{1,2} d_{2}+n_{1} \\
& y_{2}=h_{2,1} d_{1}+h_{2,2} d_{2}+n_{2} \\
& {\left[\begin{array}{l}
y_{1} \\
y_{2}
\end{array}\right]=\left[\begin{array}{ll}
h_{1,1} & h_{1,2} \\
h_{2,1} & h_{2,2}
\end{array}\right]\left[\begin{array}{l}
d_{1} \\
d_{2}
\end{array}\right]+\left[\begin{array}{l}
n_{1} \\
n_{2}
\end{array}\right]}
\end{aligned}
$$

Equation(5) indicates first received signal. $y_{1}$ and $y_{2}$ indicate received symbols to first and second antenna. And, these are expressed as matrix like equation(5) for convenience. Only transmitted signal from first transmitted antenna has to be received to first received antenna. But, looking at the equation(5), it is able to know that the transmitted signal form the others antenna was added to desired signal. By these reason, MIMO detection algorithm uses in receive part.

$$
\begin{aligned}
& y=H d+n \\
& W=\left(H^{H} H\right)^{-1} H^{H}
\end{aligned}
$$

$$
\begin{aligned}
W y & =\left(H^{H} H\right)^{-1} H^{H}(H d+n) \\
& =d+\left(H^{H} H\right)^{-1} H^{H} n
\end{aligned}
$$

Equation(6) is expressed the pseudo Inverse that is content with $\mathrm{WH}=\mathrm{I}$ in order to find transmitted signal $\mathrm{x}$. Received signal $\mathrm{y}$ is indicated that transmitted signal $\mathrm{x}$ is multiplied by channel $\mathrm{H}$ and is added noise $\mathrm{n}$. So, transmitted signal $\mathrm{x}$ is detached by multiplying the pseudo Inverse $\mathrm{W}$ and the received signal y like equation(7), this method means Zero Forcing.

$$
\begin{aligned}
& \left.E\left\{[W y-d][W y-d]^{H}\right\}\right] \\
& W=\left(H^{H} H+N_{0} I\right)^{-1} H^{H}
\end{aligned}
$$

Equation(8) is expressed the pseudo inverse of MMSE. Pseudo inverse of MMSE is similar to Zero Forcing, but $N_{0} I$ term exist in MMSE. In the case of the Zero Forcing, Pseudo Inverse is found by using estimation matrix equation. However, pseudo Inverse of MMSE is found by considering not only estimation matrix equation but also noise. Therefore, performance of MMSE is superior to Zero Forcing.

\section{Performance Evaluation}

In this paper, in Rayleigh fading channel, BER performances of CDSK system that is applied 2x2 MIMO with Zero Forcing and MMSE evaluate. Also, spreading factor is decided on 100, type of chaos map is decided on Tent map and Boss.

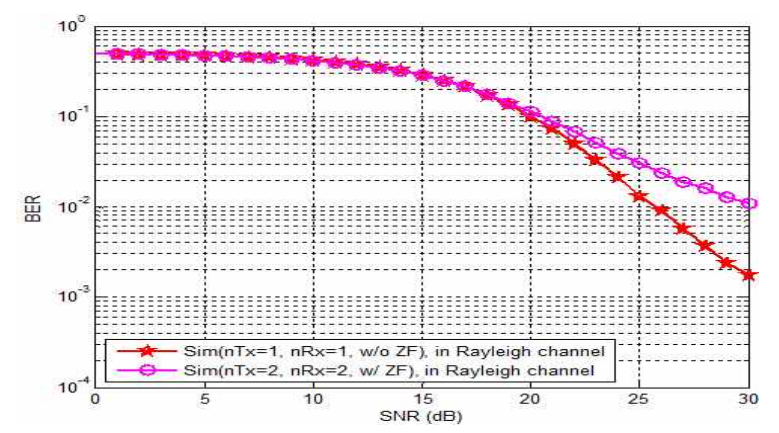

Fig. 6. BER performance of CDSK for 2x2 MIMO with Zero Forcing. 
In Rayleigh channel, Figure 6 shows BER performance of CDSK system that is applied $2 \times 2$ MIMO with Zero Forcing. Looking at the figure 6, BER performance of CDSK system that is applied $2 \times 2$ MIMO with Zero Forcing correspond to BER performance of $1 \times 1$ CDSK system until SNR(Signal-toNoise Ratio) is 20dB. However, this BER performance is evaluated badly than $1 \times 1$ CDSK system, when SNR is above $20 \mathrm{~dB}$. If pseudo inverse of Zero Forcing is multiplied to received signal, noise term like equation(7) become bigger than noise term of $1 \mathrm{x} 1$ CDSK system when SNR is above $20 \mathrm{~dB}$. So, BER performance is evaluated badly until SNR is $20 \mathrm{~dB}$.

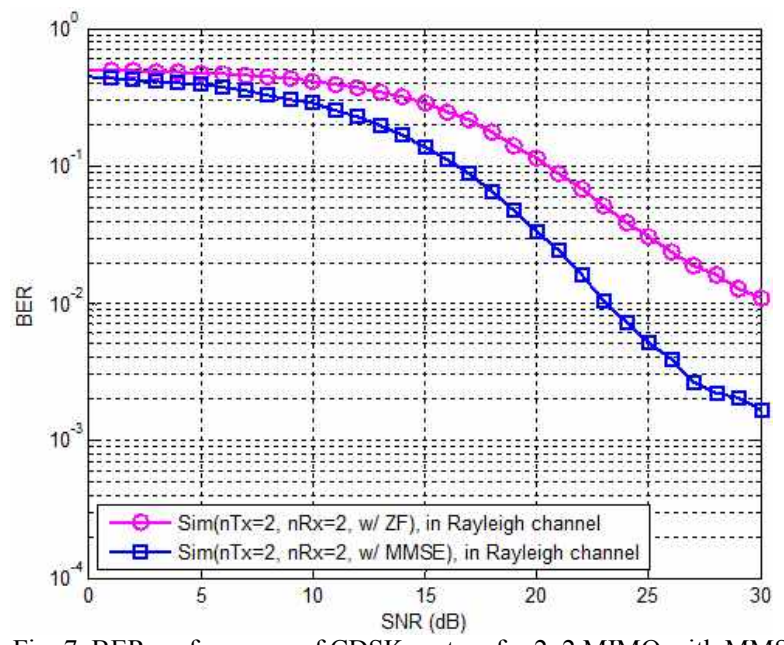

Fig. 7. BER performance of CDSK system for $2 \times 2$ MIMO with MMSE.

Figure 7 shows BER performance of CDSK system that is applied 2x2 MIMO with MMSE and Zero Forcing, and this figure compares Zero Forcing and MMSE. As MIMO detection algorithm, if MMSE is used, BER performance is superior to Zero Forcing. Because pseudo inverse of MMSE is considered not only estimation matrix equation but also noise term, and minimizes errors.

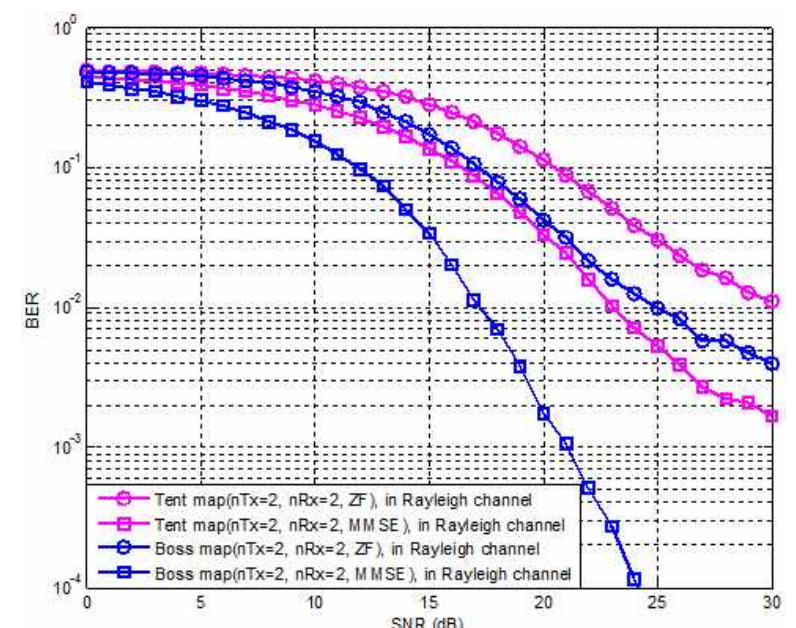

Fig. 8. BER performance of Boss map for $2 \times 2$ MIMO with Zero Forcing and MMSE.

Figure 8 shows BER performance of Boss map that is applied 2x2 MIMO with Zero Forcing and MMSE as MIMO detection algorithm. If MMSE is applied to CDSK system using Boss map, BER performance is superior to BER performance that is applied Zero Forcing. And, BER performance of Boss map is improved than Tent map is used.

\section{Conclusion}

In this paper, in Rayleigh fading channel, BER performances of 2x2 MIMO CDSK system applying MIMO detection algorithm like Zero Forcing and MMSE is evaluated. Chaos communication system has many transmitted symbols, because information signal is spread due to the characteristic of chaos map. This paper we know that in $2 \times 2$ MIMO environment this symbol is able to detach through Zero forcing and MMSE. If Zero Forcing is used as MIMO detection algorithm, BER performance of $2 \times 2$ MIMO CDSK system applying Zero Forcing correspond to BER performance of 1x1 CDSK system, when SNR is low. But as SNR rises $2 \times 2$ MIMO CDSK system applying Zero Forcing performance gets worse than $1 \times 1$ CDSK system. And, if MMSE is used as MIMO detection algorithm, BER performance is very superior to Zero Forcing. When $2 \times 2$ MIMO CDSK system using BOSS map, applying MMSE CDSK system BER performance is superior than that is applying Zero Forcing. In conclusion, MMSE performance is better than Zero Forcing in chaos communication system because pseudo Inverse of MMSE is considering not only estimation matrix equation but also noise. And, in $2 \times 2$ MIMO environment, we can know that BER performance of Boss map is better than Tent map.

\section{Acknowledgment}

This research was supported by Basic Science Research Program through the National Research Foundation of Korea(NRF) funded by the Ministry of Education, Science and Technology (No.2012017339) and (No.2013R1 A2A2A01005849)

\section{References}

[1] M. Sushchik, L.S. Tsimring and A.R. Volkovskii, "Performance analysis of correlation- based communication schemes utilizing chaos," Circuits and Systems I: Fundamental Theory and Applications, IEEE Transactions on, vol. 47, no. 12, pp. 1684-1691, Dec. 2000.

[2] Sung Il Hong and Eun Young Jang, "FPGA implementation of digital transceiver using chaotic signal," Korea Institute of Information Technology Review, vol. 8, no. 8, pp. 9-15, Aug. 2010.

[3] Q. Ding and J. N. Wang, "Design of frequency-modulated correlation delay shift keying chaotic communication system," Communications, IET, vol. 5, no. 7, pp. 901-905, May 2011.

[4] Chen YiPing, Shi Ying and Zhang Dianlun, "Performance of differential chaos-shift-keying digital communication systems over several common channels," Future Computer and Communication (ICFCC), 2010 2nd International Conference on, vol. 2, pp. 755- 759, May 2010.

[5] Suwa Kim, Junyeong Bok and Heung-Gyoon Ryu, "Performance evaluation of DCSK system with chaotic maps," Information Networking (ICOIN), 2013 International Conference on, pp. 556-559, Jan. 2013. 
[6] S. Arai and Y. Nishio, "Noncoherent correlation-based communication systems choosing different chaotic maps," Proc. IEEE Int. Symp. on Circuits and Systems, New Orleans, USA, pp. 1433-1436, June 2007.

[7] Junyeong Bok and Heung-Gyoon Ryu, "Digital chaotic communication system based on CDSK modulation," The Journal of the Korean Institute of Communications and Information Sciences, vol. 38A, no. 6, pp. 479-485, June 2013.

[8] Jun-Hyun Lee and Heung-Gyoon Ryu, "New Chaos Map for CDSK Based Chaotic Communication System," The 28th International Technical Conference on Circuit/System, Computers and Communication (ITC-CSCC 2013), Yeosu, Korea, pp. 775-778, July 2013.

[9] M.A. Ben Farah, A. Kachouri and M. Samet, "Design of secure digital communication systems using DCSK chaotic modulation," Design and Test of Integrated Systems in Nanoscale Technology, 2006. DTIS 2006. International Conference on, pp. 200-204, Sept. 2006.

[10] Jun-Hyun Lee and Heung-Gyoon Ryu, "Anti- jamming performance of chaos communications system using DCSK and CDSK modulation method," The Journal of Korean Institute of Electromagnetic Engineering and Science, vol. 24, no. 4, pp. 417-425, Apr. 2013.

[11] G. Kaddoum, Mai Vu and F. Gagnon, "On the performance of chaos shift keying in MIMO communications systems," Wireless Communications and Networking Conference (WCNC), 2011 IEEE, pp. 1432-1437, March 2011.

[12] Dian-Wu Yue and K.Q.T. Zhang, "Wireless MIMO systems in fading environments: A stabilizing transmission approach," Wireless Communications, Networking and Mobile Computing (WiCOM), 2012 8th International Conference on, pp. 1-8, Sept. 2012.

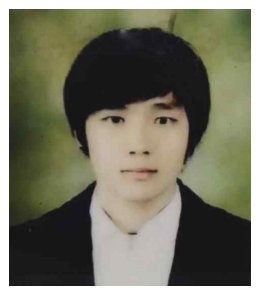

Sung-Wook Yoon was born in Daegu, Republic of Korea in 1989. He will receive the B.S degrees in the department of electronic engineering from Chungbuk National University in 2014. Now he is currently working toward B.S degree at the department of Electronic Engineering, Chungbuk National University, Republic of Korea.

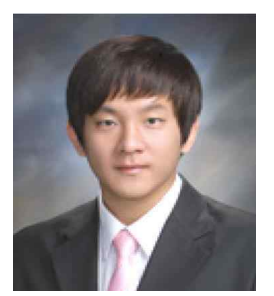

Jun-Hyun Lee was born in Ansan, Republic of Korea in 1989. He received the B.S degrees in the department of electronic engineering from Chungbuk National University in 2013. Now he is currently working toward M.S degree at the department of Electronic Engineering, Chungbuk National University, Republic of Korea.

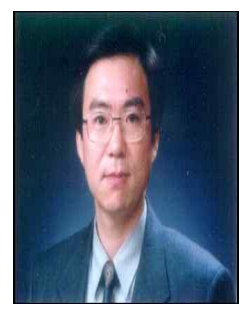

Heung-Gyoon Ryu (M'88) was born in Seoul, Republic of Korea in 1959. He received the B.S. and M.S. and Ph.D. degrees in electronic engineering from Seoul National University in 1982, 1984 and 1989. Since 1988, he has been with Chungbuk National University, Korea, where he is currently Professor of Department of Electrical, Electronic and Computer Engineering in Chungbuk National University. And he worked as Chief of RICIC (research institute of computer, information communication center) in Chungbuk National University from March 2002 to Feb 2004. His main research interests are digital communication systems, communication circuit design, spread spectrum system and communication signal processing. Since 1999, he has worked as reviewer of the IEEE transaction paper. He was a winner of '2002 ACADEMY AWARD' from the Korea Electromagnetic Engineering Society, Korea. He received the "BEST PAPER AWARD" at the 4th International Conference on Wireless Mobile Communications (ICWMC 2008) Athens, Greece, July 27-Aug.1, 2008. Also, He received the "BEST PAPER AWARD" at the International Conference on Advances in Satellite and Space Communications (SPACOMM 2009), Colmar France, July 20-25, 2009 